\title{
Complement system component dysregulation is a distinctive feature of COVID-19 disease: a prospective and comparative analysis of patients admitted to the emergency department for suspected COVID-19 disease
}

\author{
Nadine Gauchel ${ }^{1,2} \cdot$ Marina Rieder $^{1,2} \cdot$ Krystin Krauel $^{2} \cdot$ Isabella Goller $^{1,2} \cdot$ Maren Jeserich $^{1,2} \cdot$ Ulrich Salzer $^{3}$. \\ Ana Cecilia Venhoff ${ }^{3}$. Niklas Baldus ${ }^{1,2}$ • Luisa Pollmeier ${ }^{4} \cdot$ Luisa Wirth $^{4}$ - Winfried Kern ${ }^{5} \cdot$ Siegbert Rieg $^{5}$. \\ Hans-Jörg Busch ${ }^{6} \cdot$ Maike Hofmann $^{7}$. Christoph Bode ${ }^{1,2} \cdot$ Daniel Duerschmied $^{1,2} \cdot$ Achim Lother $^{1,2,3}$. \\ Lukas A. Heger ${ }^{1,2}$ (D)
}

Accepted: 24 November 2021 / Published online: 14 December 2021

(c) The Author(s) 2021

\begin{abstract}
The complement system (CS) plays a pivotal role in Coronavirus disease 2019 (COVID-19) pathophysiology. The objective of this study was to provide a comparative, prospective data analysis of CS components in an all-comers cohort and COVID19 patients. Patients with suspected COVID-19 infection admitted to the Emergency department were grouped for definite diagnosis of COVID-19 and no COVID-19 accordingly. Clinical presentation, routine laboratory and von Willebrand factor (vWF) antigen as well as CS components 3, 4 and activated 5 (C5a) were assessed. Also, total complement activity via the classical pathway (CH50) was determined. Levels of calprotectin in serum were measured using an automated quantitative lateral flow assay. We included 80 patients in this prospective trial. Of those $19(23.7 \%)$ were tested positive for severe acute respiratory syndrome coronavirus 2 (SARS-CoV-2). Patients with COVID-19 had higher levels of CS components 5a and $4(54.79$ [24.14-88.79] ng/ml vs. 35 [23.15-46.1] ng/ml; $\mathrm{p}=0.0433$ and 0.3772 [ \pm 0.1056$] \mathrm{g} / \mathrm{L}$ vs. 0.286 [0.2375-0.3748] $\mathrm{g} / \mathrm{L} ; \mathrm{p}=0.0168)$. COVID-19 patients had significantly higher levels of vWF antigen when compared to the control group (288.3 [ \pm 80.26$] \%$ vs. 212 [151-320] \%; p=0.0469). There was a significant correlation between CS C3 and 5a with vWF antigen $\left(r_{s}=0.5957[p=0.0131]\right.$ and $\left.r_{s}=0.5015[p=0.042]\right)$ in COVID-19 patients. There was no difference in calprotectin plasma levels $(4.786[ \pm 2.397] \mu \mathrm{g} / \mathrm{ml}$ vs. $4.233[ \pm 2.142] \mu \mathrm{g} / \mathrm{ml} ; \mathrm{p}=0.4175)$ between both groups. This prospective data from a single centre all-comers cohort accentuates altered levels of CS components as a distinct feature of COVID-19 disease. Deregulation of CS component 3 and C5a are associated with increased vWF antigen possibly linking vascular damage to alternative CS activation in COVID-19.
\end{abstract}

Keywords COVID-19 · Complement components · Coagulopathy · Von Willebrand factor · Calprotectin

Lukas A. Heger

lukas.heger@uniklinik-freiburg.de

1 Department of Medicine III (Interdisciplinary Medical Intensive Care), Medical Center, Faculty of Medicine, University of Freiburg, Freiburg, Germany

2 Department of Cardiology and Angiology I, Heart Center, University of Freiburg, Hugstetter Strasse 55, 79106 Freiburg, Germany

3 Department of Rheumatology and Clinical Immunology, University Hospital of Freiburg, Freiburg, Germany

4 Institute of Experimental and Clinical Pharmacology and Toxicology, Faculty of Medicine, University of Freiburg, Freiburg, Germany
5 Division of Infectious Diseases, Department of Medicine II, Medical Center, Faculty of Medicine, University of Freiburg, University of Freiburg, Freiburg, Germany

6 Department of Emergency Medicine, Faculty of Medicine, University Hospital of Freiburg, University of Freiburg, Freiburg, Germany

Department of Medicine II, Faculty of Medicine, University Hospital Freiburg, University of Freiburg, Freiburg, Germany 


\section{Abbreviations}

COVID-19 Coronavirus disease 2019

CS Complement System

ED Emergency department

SARS-CoV-2 Severe acute respiratory syndrome coronavirus 2

VWF

\section{Highlights}

- Complement system (CS) component dysregulation is a distinctive feature of COVID-19 disease.

- Patients with COVID-19 have higher levels of CS components $5 \mathrm{a}$ and 4.

- Elevated levels of CS component 5a correlate with elevated levels of von Willebrand Factor antigen in COVID19 patients.

- Calprotectin plasma levels do not differ between COVID19 patients and emergency department all-comers.

\section{Introduction}

The complement system (CS), as part of the innate immune response, plays a profound role in coordinating the inflammatory response to pathogens and its unrestrained activation has been implicated in the pathogenesis of Coronavirus disease 2019 (COVID-19) [1-3]. Multiple studies have shown increased levels of CS components in patients with COVID-19 as well as deposition of activated complement proteins in injured organs $[3,4]$. Recently a severe acute respiratory syndrome coronavirus 2 (SARS-CoV-2) binding site affecting a CS activating serine-protease was discovered as possible target for therapy [5]. Understanding CS activation in COVID-19 could provide insights into the pathogenesis of hypercoagulability and increased risk for coagulopathic events, both hallmarks of COVID-19 disease [4, 6-8].

The relevance of COVID-19-coagulation abnormalities remains of pivotal importance as a substantial proportion of patients with mild COVID-19 is developing, sometimes unrecognized, thromboembolic complications [9]. More specific targets are needed to optimize treatment strategies. Currently several inhibitors of the CS are in clinical trials for COVID-19 treatment (C3: NCT04395456; C5: NCT04355494 and C5a: NCT04346797) [10].

The CS is activated via different pathways [10-12]. One of this pathways seems to be via endothelial cell dysfunction and injury [13]. Von Willebrand factor antigen has been suggested as marker of endothelial dysfunction in vascular diseases before and imbalance of the VWF-axis has been implicated in COVID-19 disease [14, 15].
Several other markers are suggested for COVID-19 prognosis and diagnosis as well [16]. Calprotectin a member of the S100 family has recently gained increasing attention as a potential novel biomarker of inflammatory disorders [17]. Despite lacking consensus on measurement of calprotectin levels several studies report significant differences in calprotectin plasma levels in COVID-19 disease [18, 19]. However, little is known of the diagnostic capacities of calprotectin in emergency department patients during the COVID-19 pandemic.

\section{Methods}

\section{Study design}

We here report data from an investigator-initiated, singlecenter prospective registry study to evaluate biomarkers associated with COVID-19 (DRKS00021206, Deutsches Register klinische Studien (DRKS)) conducted at the University Medical Center-University of Freiburg.

The protocol of this study conforms to the ethical guidelines of the 1975 Declaration of Helsinki and was approved by the institutional ethical committee of the University of Freiburg (EK 153/20).

\section{Study population}

All-comers admitted to the department of emergency medicine of the University Medical Center-University of Freiburg between 26th of March 2020 and the 22th of May 2020 with suspected COVID-19 were included in this prospective trial. The decision to perform a PCR-test for severe acute respiratory syndrome coronavirus 2 (SARS-CoV-2) was made independently of study inclusion by the treating physician and patients were asked to participate before the test results were available.

Written informed consent was obtained from all patients prior to study inclusion.

Patients with a positive PCR-test for SARS-CoV-2 were finally allocated to the "positive" COVID-19 group, patients with a negative PCR-test for SARS-CoV-2 to the control group (Fig. 1). No included patient was vaccinated against SARS-CoV-2.

\section{Study plan}

If patients had agreed to participate, overall characteristics such as sex, age, body-mass-index (BMI), medical history, laboratory parameters, clinical symptoms or previous medication were recorded. 


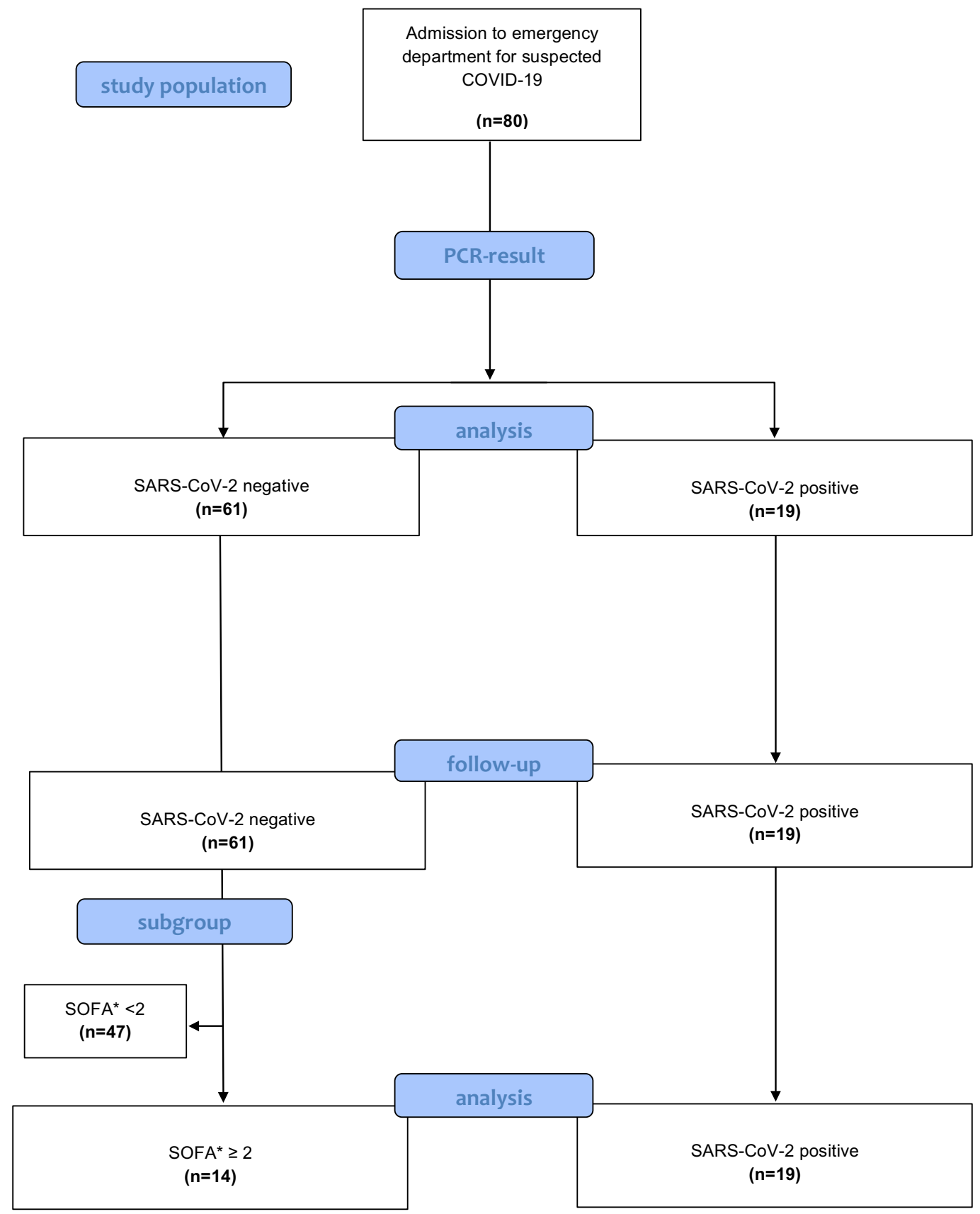

Fig. 1 Schematic representation of the allocation to the positive or negative group of the 80 participants. The flow diagram is based on the template of the CONSORT flow diagram. (38) *SOFA Sequential Organ Failure Assessment

\section{Endpoint}

The primary end-point was diagnosis of COVID-19.

\section{Complement system components}

The concentration of complement factors C3 and C4 in serum were determined by nephelometry on an Atellica NEPH 630 System (Siemens Healthcare GmbH, Erlangen, Germany) using standard settings and assays as recommended by the manufacturer. Semiquantitative assessment of functional classical complement pathway activation 
(CH50) in serum was performed with the Wieslab Complement system Classical pathway ELISA kit (SVAR LIFE SCIENCEAB, Malmö, Sweden), following the instructions given by the manufacturer.

Component C5a level in serum was determined by a commercially available ELISA kit (DuoSet, R\&D Systems), according to the manufacturer's instructions.

\section{Calprotectin}

Calprotectin (MRP8/14 or S100A8/A9) in serum was measured using Quantum Blue sCAL reagents (BÜHLMANN Laboratories AG, Schönenbuch, Switzerland) on a Quantum Blue Reader 2nd generation (BÜHLMANN Laboratories AG, Schönenbuch, Switzerland) using standard settings and protocols as recommended by the manufacturer.

\section{von Willebrand factor \& Serotonin}

Von Willebrand factor antigen and activity were measured using Sysmex CS-5100 System ${ }^{\mathrm{TM}}$ (Siemens Healthcare Diagnostics, Erlangen, Germany) according to the manufacturer's instructions. Reagents used on the CS-5100/ analyzers were INNOVANCE VWF Ac/Standard Human Plasma/ Control Plasma N, Control Plasma P (Catalogue No.: OPHL03 Siemens Healthcare Diagnostics Products $\mathrm{GmbH}$ ) for vWF activity and von Willebrand Antigen/ Standard Human Plasma/ Control Plasma N, Control Plasma P (Catalogue No.: OPAB03 Siemens Healthcare Diagnostics Products $\mathrm{GmbH}$ ) for vWF antigen.

Serotonin was analyzed in serum samples using an enzymatic immunoassay kit (Serotonin ELISAFastTrack, LDN, Nordhorn, Germany) according to the manufacturer's instructions. All samples and standards were analyzed on a microplate reader set at $450 \mathrm{~nm}$.

\section{Data analysis}

For analysis, data were blinded to patient identity. Statistical analyses were performed using SPSS (version 25, IBM, SPSS Statistics, Armonk, USA) and GraphPad Prism 9 (GraphPad Software, San Diego, USA).

Variables following Gaussian distribution were compared using student's t-test, non-normally distributed continuous values by using Mann-Whitney-U test. Categorical variables were assessed by chi-square test or Fisher's exact test as appropriate.

A two-tailed p-value less than 0.05 was considered statistically significant. Simple linear regression analysis was used to model the relationship between two complement components and von Willebrand Factor.

Data are presented as mean \pm standard deviation if found to follow a Gaussian distribution or otherwise as median with interquartile range. Spearman's correlation for non parametric data was used to measure the strength and direction of monotonic association between CS components and vWF antigen.

\section{Follow-up}

Patients were contacted 1 month after discharge to assess whether they had persistent incapacity to work.

\section{Results}

\section{Baseline characteristics}

We included 80 patients admitted to ED for respiratory failure in this prospective cohort study. Of those 19 (23.7\%) were tested positive for SARS-CoV-2 and henceforth allocated to the COVID-19 group. In the COVID-19 cohort, 50,8\% were male and in the control group $40 \%$ $(\mathrm{p}=0,4)$. Patients in the non-COVID-19 control group were age matched $(62.9[ \pm 19.6]$ years vs. $58.9[ \pm 14.2]$ years; $p=0.4074)$ with the COVID-19 group. In the nonCOVID-19 group, 4 (6.6\%) had symptomatic anemia, 9 (14.8\%) patients had pneumonia, 9 (14.8\%) patients were hospitalized for cardiac decompensation, 4 (6.6\%) had pulmonary embolism, 16 (26.2) patients hat urogenital or gastrointestinal infections and $11(18 \%)$ various forms of cancer (including fever in neutropenia). (Supplement Fig. 1).

Patients with COVID-19 had similar Body Mass Index when compared to the control group (24.54 [21.9-27.17] $\mathrm{kg} / \mathrm{m}^{2}$ vs. $\left.25.9[ \pm 5.1] \mathrm{kg} / \mathrm{m}^{2} ; \mathrm{p}=0.4414\right)$.

There was no significant difference in history of coronary artery disease $(13.1 \%$ vs $10 \%$; $\mathrm{p}=0.133)$, hypertension $(50.1 \%$ vs. $30 \% ; \mathrm{p}=0.1048)$ or diabetes mellitus ( $14.8 \%$ vs. $10 \% ; \mathrm{p}=0.5902$ ) between the control group and COVID-19 patients. COVID-19 patients were more likely to be on angiotensin II receptor blockers (35\% vs. 13.3\%; $\mathrm{p}=0.0316$ ). (Table 1) Nobody in the COVID-19 group and $4(6 \%)$ patients in the control-group were on medication with potassium-sparing diuretics.

\section{Laboratory data \& characteristics at admission}

COVID-19 patients were more likely to present with fever ( $80 \%$ vs. $49.2 \%$; $p=0.0158)$, cough ( $65 \%$ vs. $37.5 \%$; $\mathrm{p}=0.033)$ with a tendency for dyspnoea $(57.4 \%$ vs. $35 \%$; $\mathrm{p}=0.0822$ ) when compared to non-COVID-19 patients. There was no difference in the Visual Analogue Scales (VAS) for subjective disease severity between both groups (4.6 [ \pm 2.8$]$ vs. $5.4[ \pm 2.9] ; p=0.3489)$. At admission there was no statistical difference between the control group and patients with COVID-19 in respect to mean arterial pressure 
Table 1 Baseline characteristics at admission

\begin{tabular}{|c|c|c|c|c|c|}
\hline \multirow{2}{*}{$\frac{\text { Variable }}{\text { Age (years) }}$} & \multicolumn{2}{|c|}{ Non-COVID $19(n=61)$} & \multicolumn{2}{|c|}{ COVID-19 $(n=19)$} & \multirow{2}{*}{$\frac{\text { P-value }}{0.4074^{\mathrm{a}}}$} \\
\hline & 62.9 & $( \pm 19.6)$ & 58.9 & $( \pm 14.2)$ & \\
\hline Body Mass Index & 24.54 & $(21.9-27.17)$ & 25.9 & $( \pm 5.1)$ & $0.4414^{\mathrm{b}}$ \\
\hline Mean arterial pressure $(\mathrm{mmHg})$ & 99 & $( \pm 16.2)$ & 99.3 & $( \pm 11.84)$ & $0.9413^{\mathrm{a}}$ \\
\hline Heart Rate (beats/minute) & 88 & $( \pm 19.8)$ & 90.2 & $( \pm 15.7)$ & $0.6769^{\mathrm{a}}$ \\
\hline Respiratory rate/minute & 23 & $(18.3-30)$ & 25.3 & $( \pm 9.1)$ & $0.8893^{\mathrm{b}}$ \\
\hline O2-Saturation (\%) & 96 & (94-98) & 96 & $(93.598)$ & $0.7234^{\mathrm{b}}$ \\
\hline Temperature (in ${ }^{\circ} \mathrm{C}$ ) & 37.5 & $( \pm 0.99)$ & 37.5 & $( \pm 0.83)$ & $0.846^{\mathrm{a}}$ \\
\hline SOFA* Score & 1 & $(0-2)$ & 0.5 & $(0-1.75)$ & $0.6926^{\mathrm{b}}$ \\
\hline Leukocytes $(\mathrm{Tsd} / \mu \mathrm{l})$ & 8.9 & $(6-13.6)$ & 4.7 & $( \pm 2.31)$ & $<0.0001^{\mathrm{b}}$ \\
\hline Haemoglobin $(\mathrm{g} / \mathrm{dl})$ & 11.41 & $( \pm 2.78)$ & 12.7 & $( \pm 2.5)$ & $0.0691^{\mathrm{a}}$ \\
\hline Creatinin $(\mathrm{mg} / \mathrm{dl})$ & 0.97 & $(0.77-1.4)$ & 0.92 & $(0.72-1.2)$ & $0.3405^{\mathrm{b}}$ \\
\hline C-reactive protein $(\mathrm{mg} / \mathrm{l})$ & 29.30 & $(4.52-79.9)$ & 46.8 & $( \pm 56.8)$ & $0.405^{\mathrm{b}}$ \\
\hline Procalcitonin $(\mathrm{ng} / \mathrm{ml})$ & 0.08 & $(0.05-0.35)$ & 0.07 & $(0.06-0.15)$ & $0.5378^{\mathrm{b}}$ \\
\hline Aspartat-Aminotransferase (U/I) & 25 & $(18.8-37)$ & 34.5 & $(28.5-69.8)$ & $0.0046^{\mathrm{b}}$ \\
\hline PTT at admission (sec.) & 31 & $(28-34)$ & 29.8 & $( \pm 3)$ & $0.2947^{\mathrm{b}}$ \\
\hline Laktatdehydrogenase (U/I) & 234.5 & $(192-295.5)$ & 249 & $(191-341)$ & $0.4543^{\mathrm{b}}$ \\
\hline Creatinkinase (U/I) & 68.5 & $(48.8-119.5)$ & 100.3 & $( \pm 62.6)$ & $0.6168^{\mathrm{b}}$ \\
\hline D-Dimere & 1.15 & $(0.34-2.13)$ & 1.13 & $(0.47-1.98)$ & $0.9087^{\mathrm{b}}$ \\
\hline Calprotectin $(\mu \mathrm{g} / \mathrm{ml})$ & 4.786 & $( \pm 2.397)$ & 4.233 & $( \pm 2.142)$ & $0.4175^{\mathrm{a}}$ \\
\hline Pro-BNP** (pg/ml) & 318 & $(81.5-3247)$ & 106 & $(50-404)$ & $0.0211^{\mathrm{b}}$ \\
\hline Length of hospital stay (days) & 7 & $(2.75-15)$ & 12.25 & $( \pm 9.8)$ & $0.2217^{\mathrm{b}}$ \\
\hline Sex (Male in \%) & 50.8 & & 40 & & $0.4^{\mathrm{c}}$ \\
\hline Visual analogue scales (VAS) & 4.6 & $( \pm 2.8)$ & 5.4 & $( \pm 2.9)$ & $0.3489^{\mathrm{a}}$ \\
\hline Cough $(\%)$ & 37.7 & & 65 & & $0.033^{\mathrm{c}}$ \\
\hline Dyspnea (\%) & 57.4 & & 35 & & $0.0822^{\mathrm{c}}$ \\
\hline Fever $(\%)$ & 49.2 & & 80 & & $0.0158^{\mathrm{c}}$ \\
\hline Angiotensin II receptor blockers (\%) & 13.3 & & 35 & & $0.0316^{\mathrm{c}}$ \\
\hline Coronary Artery Disease (\%) & 13.1 & & 10 & & $0.7133^{\mathrm{c}}$ \\
\hline Hypertension (\%) & 50.1 & & 30 & & $0.1048^{\mathrm{c}}$ \\
\hline Diabetes Mellitus (\%) & 14.8 & & 10 & & $0.5902^{\mathrm{c}}$ \\
\hline History or current smoking (\%) & 52.5 & & 50 & & $0.8485^{\mathrm{c}}$ \\
\hline
\end{tabular}

*SOFA Sequential Organ Failure Assessment (SOFA) Score, **BNPB-type natriuretic peptide; Variables following Gaussian distribution were compared using student's t-test (a), non-normally distributed continuous values by using Mann-Whitney-U test (b). Categorical variables were assessed by chi-square test or Fisher's exact test as appropriate (c)
$(99[ \pm 16.2] \mathrm{mmHg}$ vs. $99.3[ \pm 11.84] \mathrm{mmHg} ; \mathrm{p}=0.9413)$, heart rate $(88[ \pm 19.8]$ beats/minute vs. $90.2[ \pm 15.7]$ beats/ minute; $\mathrm{p}=0.6769)$, respiratory rate $(23[18.3-30]$ per minute vs. $25.3[ \pm 9.1]$ per minute; $p=0.8893)$ or difference in saturation at admission (96 [94-98] \% vs 96 [93.5-98]; $\mathrm{p}=0.7234$ ).

There was no statistically significant difference in severity of disease assessed by SOFA Score between patients with COVID-19 and the control group $(0.5$ [0-1.75] vs. 1 [0-2]; $\mathrm{p}=0.6926$ ).

Non-COVID-19 patients had higher blood levels of leukocytes $(8.9[6-13.6] \mathrm{K} / \mu \mathrm{l}$ vs. $4.7[ \pm 2.31] \mathrm{K} / \mu \mathrm{l} ; \mathrm{p}=<0.0001)$ and pro-BNP (318 [81.5-3247] pg/ml vs. 106 [50-404] $\mathrm{pg} / \mathrm{ml} ; \mathrm{p}=0.0211$ ) when compared to COVID-19 patients. There was no difference in C-reactive protein (29.30
[4.52-79.9] mg/l vs. 46.8 [ \pm 56.8$] \mathrm{mg} / \mathrm{l} ; \mathrm{p}=0.405)$ or procalcitonin $(0.08$ [0.05-0.35] ng/ml vs. 0.07 [0.06-0.15] ng/ $\mathrm{ml} ; \mathrm{p}=0.5378$ ) between the control-group and the COVID19 group.

COVID-19 patients had significantly higher blood levels of aspartat-aminotransferase when compared to the control group (34.5 [28.5-69.8] U/I vs. 25 [18.8-37] U/I; $\mathrm{p}=0.0046$ ).

There was no difference between calprotectin plasma levels between patients of the control group and patients with COVID-19 $(4.786( \pm 2.397) \mu \mathrm{g} / \mathrm{ml}$ vs. $4.233( \pm 2.142) \mu \mathrm{g} /$ $\mathrm{ml} ; \mathrm{p}=0.4175)$. 
Fig. 2 Comparative analysis of Complement factors (a Complement component $5 \mathrm{a}$ in $\mathrm{ng} / \mathrm{ml}$; b Complement component 3 in $\mathrm{g} / \mathrm{L} ; \mathbf{c}$ Complement component 4 in $\mathrm{g} / \mathrm{L} ; \mathbf{d} \mathrm{CH} 50(\%)$ in patients hospitalized for respiratory failure and COVID-19 respectively. Data are presented as scatter block with median and interquartile range
Fig. 3 Comparative analysis of plasma serotonin $\mathbf{a}$ and platelet count $\mathbf{b}$ in patients hospitalized for respiratory failure and COVID-19 respectively. Data are presented as scatter block with median and interquartile range a

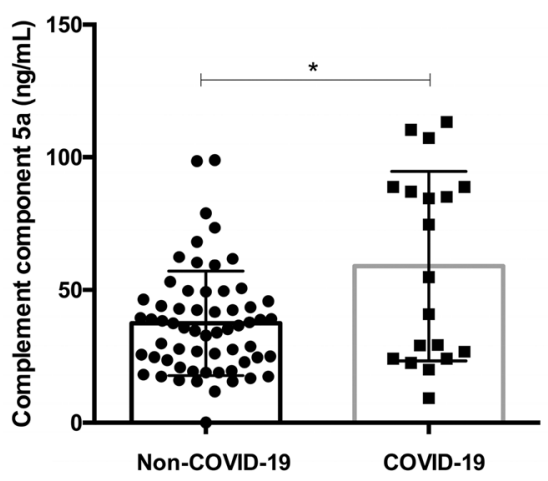

C

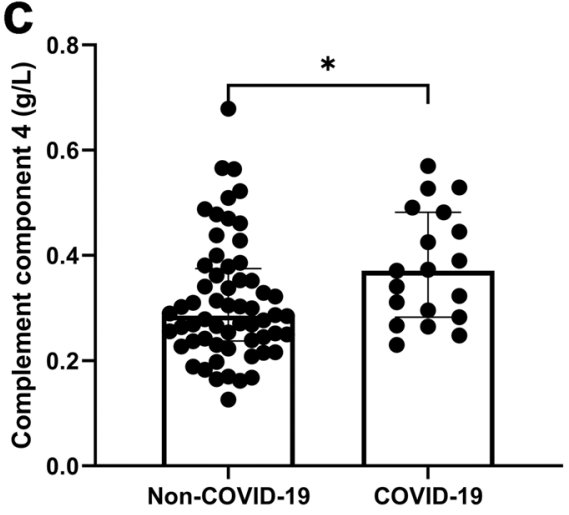

2

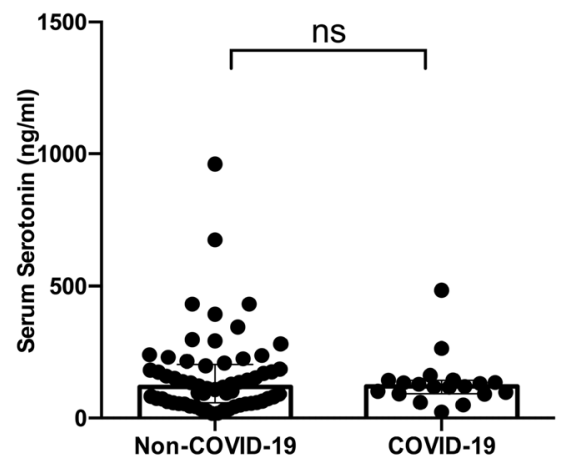

b

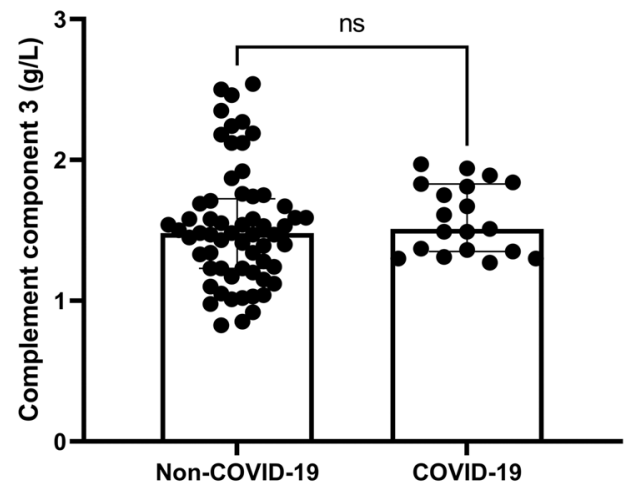

d

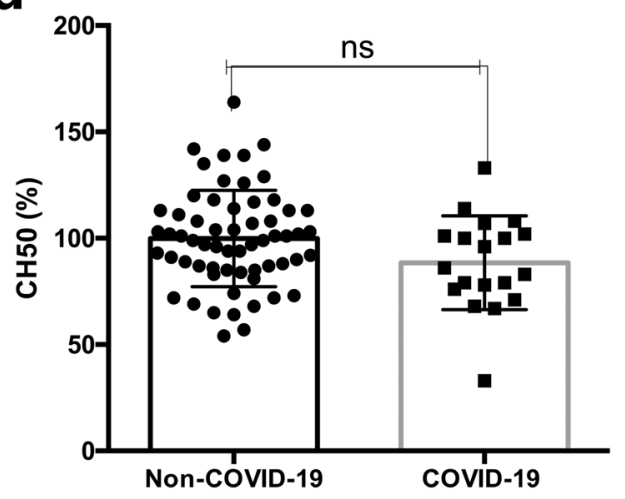

b

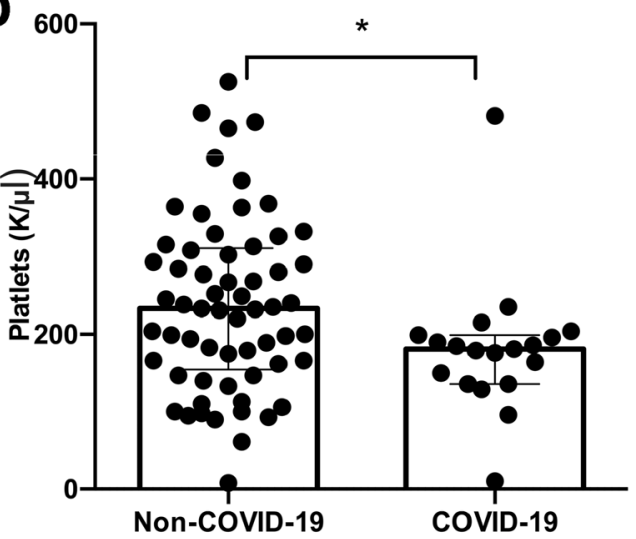

\section{Complement \& complement activation}

Patients with COVID-19 had statistically significant higher levels of complement components 5a and 4 (54.79 [24.14-88.79] $\mathrm{ng} / \mathrm{ml}$ vs. 35 [23.15-46.1] ng/ml; $\mathrm{p}=0.0433$ and $0.3772[ \pm 0.1056]$ vs. 0.286 [0.2375-0.3748]; $\mathrm{p}=0.0168$ ). There was no significant difference in classical CS activation or CS C3 levels between COVID-19 patients and the control group (CH50: $88.47[ \pm 22.1] \%$ vs. 99.85 $[ \pm 22.6] \% ; \mathrm{p}=0.06 ; \mathrm{C} 3: 1.51[1.35-1.83] \mathrm{g} / \mathrm{L}$ vs. 1.53 $[ \pm 0.4252] \mathrm{g} / \mathrm{L} ; \mathrm{p}=0.3049$ ). (Fig. 2).
Patients with COVID-19 had lower platelet count when compared to the control group (180 [136-198.3] K/ul vs. 237.1 [ \pm 91.73$] \mathrm{K} / \mathrm{ul} ; \mathrm{p}=0.0304)$. There was no statistically significant difference in serotonin plasma levels between the COVID-19 cohort and the control group (109.5 [ \pm 35.98$]$ ng/ml vs. 116 [58.6-201.3] ng/ml; $\mathrm{p}=0.6$ ). (Fig. 3).

Patients with COVID-19 had significantly higher levels of von Willebrand Factor antigen when compared to the control group $(288.3[ \pm 80.26] \%$ vs. $212[151-320] \%$; $p=0.0469)$. There was no difference in von Willebrand factor activity 
Fig. 4 Comparative analysis of plasma levels of von Willebrand factor antigen $\mathbf{a}$ and von Willebrand factor activity $\mathbf{b}$ in patients hospitalized for suspected COVID-19 and definite diagnosis of COVID-19 respectively. Data are presented as scatter block with median and interquartile range

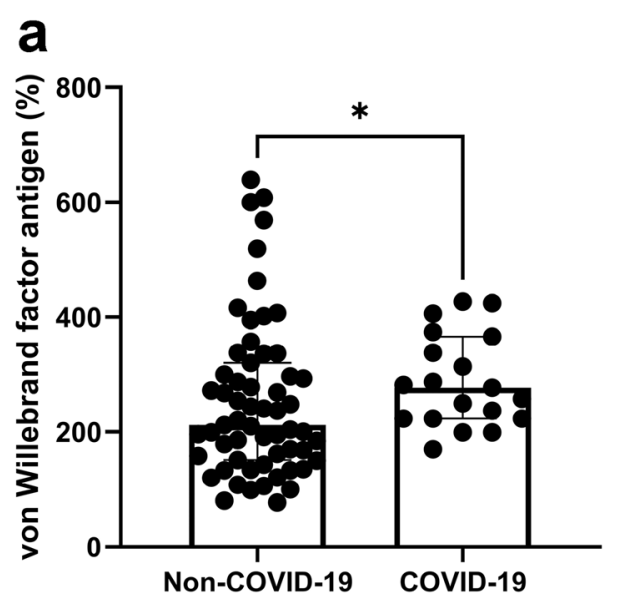

b

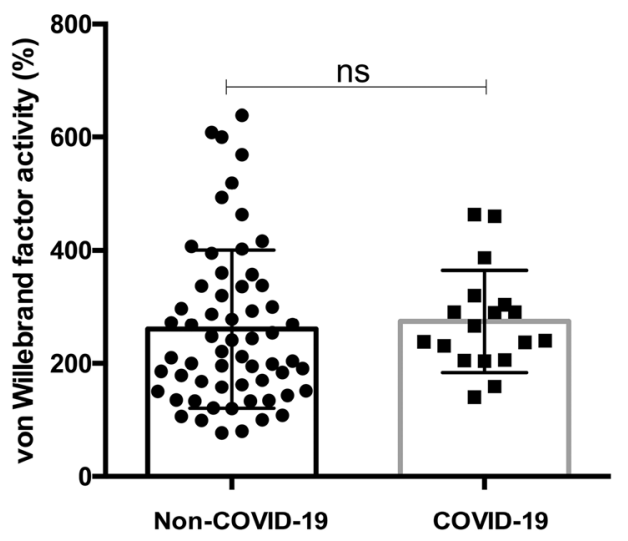

between the control group and COVID-19 patients (147 [ \pm 129.6$] \%$ vs. 166 [106-220] \%; $p=0.0469)$. (Fig. 4).

There was a significant correlation between CS C 3 and $5 \mathrm{a}$ with vWF antigen $\left(r_{s}=0.5957[p=0.0131]\right.$ and $r_{s}=0.5015$ $[\mathrm{p}=0.042]$ ) in COVID-19 patients.

In a subgroup analysis comparing non-COVID-19 patients with SOFA Score $\geq 2(n=14)$ to patients with COVID-19 statistical analysis showed increased levels of complement component $3(1.321[ \pm 0.2804] \mathrm{g} / \mathrm{l}$ vs. $1.51[1.35-1.83] \mathrm{g} / \mathrm{l} ; \mathrm{p}=0.031), 4(0.2673[ \pm 0.0797]$ $\mathrm{g} / \mathrm{l}$ vs. $0.3772[ \pm 0.1056] \mathrm{g} / \mathrm{l} ; \mathrm{p}=0.0027)$ and $5 \mathrm{a}(0.159$ [0.1175-0.203] pg/ml vs. 0.493 [0.139-0.583] pg/ml; $\mathrm{p}=0.0378$ ). (Supplement Fig. 2).

\section{Characteristics of hospital stay \& follow-up}

There was no statistically significant difference in length of hospital stay(7 [2.75-15] days vs. 12.25 [ \pm 9.8$]$ days; $\mathrm{p}=0.2217$ ) between the control group and COVID-19 patients. None of COVID-19 patients and $6(9.8 \%)$ of nonCOVID-19 patients were referred to the intensive care unit. In patients with COVID-19, $10(52.6 \%)$ patients were employed prior to hospitalization as against 12 (19.7\%) patients in the non-COVID-19 control group. At one-month follow-up, significantly more patients from the COVID19 cohort were still incapacitated for work (70\% vs. $25 \%$; $\mathrm{p}=0.0348$ ).

\section{Discussion}

In this single centre prospective real world data analysis we evaluate the CS in COVID-19 disease in respect to an all comers cohort admitted to the ED. Our data implicate a derailed CS with overexpression of CS components in COVID-19 patients plasma as a distinctive feature of COVID-19 disease [20].
Several studies have described the crosstalk between CS and coagulation in sepsis [21]. Our data add to the amounting evidence, that the pro-thrombotic state in COVID-19 disease is in a similar more profound way fuelled by a derailed CS [2-4, 21, 22]. Interestingly our data shows C4 and C5a elevation but no difference in $\mathrm{C} 3$ expression. This could be in line with recent experimental data suggesting a "C3 bypass" activation of C5 by surface-deposited C4 [23].

In clinical practice, especially activated CS component $5 \mathrm{a}$ is considered to be a critical determinant of neutrophil recruitment and activation in thrombosis and early dampening could have the potential to reduce leukocyte accumulation, thrombus initiation and propagation [10, 24, 25]. However, mechanisms mediating $\mathrm{C} 3 \mathrm{a} / \mathrm{C} 5 \mathrm{a}$ generation during a pro-thrombotic state are poorly understood. Endotheliopathy as a hallmark of septic patients with injured endothelial cells provides a scaffold for coagulation [26]. Correspondingly, we report increased plasma levels of von Wilebrand factor antigen as biomarker pointing to vascular injury in COVID19 patients [13, 27, 28]. This increase in vWF antigen was associated with CS components linking vWF antigen to CS alteration.

Correspondingly, recent data show, that complement activation and endothelial perturbation parallel COVID19 severity [29]. Our prospective data from an all-comers cohort accentuates $\mathrm{VWF}$ antigen as a distinct feature of COVID-19 and add to the amounting evidence linking activation of CS with vWF antigen and endothelial damage [12, 13]. Several publications indicate alternative pathways of $C S$ activation in COVID-19 to be predominant [29, 30]. Platelets are an important modulator of CS and secrete serotonin upon activation [31, 32]. With the majority of peripheral serotonin stored in platelets, we hypothesized that activation of platelets and subsequent clearance from the blood stream could be the cause of COVID-19 thrombocytopenia and would result in increased plasma levels of serotonin. However, this was not the case. This could be partially 
explained by recent evidence showing, that increased platelet activation is a hallmark of mainly severe COVID-19 [33]. Nevertheless, platelets are chief effector cells in thrombosis and our data confirm a deregulation in platelet homeostasis in COVID-19. The exact mechanism of platelets in the pathogenesis of COVID-19 however, remains elusive.

Several studies have evaluated biomarkers that can help predict severe complications in COVID-19. Recently, calprotectin a member of the $\mathrm{S} 100$ family has gained increasing attention as a potential novel biomarker of inflammatory disorders [17]. Despite lacking consensus on measurement of calprotectin levels several studies report significant differences in calprotectin plasma levels in COVID-19 [18, 19]. Our prospective data analysis showed no difference in calprotectin levels at admission. Whether calprotectin may serve as predictor of disease progression is yet to be established.

Baseline laboratory parameters are suggested in COVID19 diagnosis because of their cost effectiveness and easy accessibility. Interestingly, we show no difference in D-Dimers between the two cohorts at admission [34]. This is in line with recent evidence suggesting dynamic changes in D-Dimers over the course of hospitalization to predict subsequent coagulopathy in COVID-19 patients [35].

Recently a meta-analysis isolated a pattern of abnormal liver enzymes in COVID-19 [36]. Our data confirm, that intensive monitoring for liver injury may be needed in cases with COVID-19.

While there was no subjective difference in Vision Analogue Scale for disease severity at admission and no difference in length of hospital stay our data clearly indicate the relevance of the post-acute COVID-19 syndrome and its influence on the economic fallout of the pandemic with an increased proportion of COVID-19 patients being incapacitated for work after one month. Also we show, that typical symptoms of COVID-19 including cough, fever and dyspnoea are reliable and decisive in ED triage and can help to optimize patient flow especially with increasing numbers of hospital admission [37].

\section{Limitations}

We report data from a single centre study. Larger clinical trials are needed. Complement levels are very dynamic and are result of constant synthesis and decay and especially for activation markers like $\mathrm{C} 5 \mathrm{a}$ the preanalytical handling (=the quality of the collected sample) is crucial. While we tried to establish immediate analysis blood sample collection was part of the routine process and therefore subject to inter-individual differences. Also, only a small fraction of all CS factors have been quantified by us. There are many others, some of which may be also increased or decreased in COVID-19 patients and still correlate with the disease status. Finally, since we did not collect cycle thresholds of initial SARS-CoV-2 polymerase chain reaction tests of included patients at the time point of collecting the blood sample, patients could be in different stages of the disease.

\section{Conclusion}

This prospective comparative data from a single centre allcomers cohort accentuates dysregulation of CS components as a distinct marker for COVID-19 disease. Highlighting the importance of alternative CS activation pathways through endothelial damage we show an association of CS components and von Willebrand factor antigen in COVID-19 patients. Our data provides evidence, that despite deregulation in platelet homeostasis leading to thrombocytopenia in COVID-19 patients, serum levels of serotonin are not increased. Also we show, that calprotectin, a promising marker of disease progression is of limited use in COVID19 assessment in emergency evaluation. Finally, our data adds to the understanding of the socio-ecological fallout of the COVID-19 pandemic showing a higher rate of prolonged incapacity for work in post-COVID-19 patients.

Supplementary Information The online version contains supplementary material available at https://doi.org/10.1007/s11239-021-02617-x.

Acknowledgements NG, KK, DD and AL are members of SFB1425, funded by the Deutsche Forschungs-gemeinschaft (DFG, German Research Foundation)-Project \#422681845. This work was supported by the DFG, German Research Foundation (Research Fellowship KR 4945/2-1 to K. Krauel and HE 8679/1-1:1 to L. A. Heger)

Funding Open Access funding enabled and organized by Projekt DEAL. This article is funded by Deutsche Forschungsgemeinschaft,KR 4945/2-1,Krystin Kraul,HE 8679/1-1:1,Lukas Heger.

Open Access This article is licensed under a Creative Commons Attribution 4.0 International License, which permits use, sharing, adaptation, distribution and reproduction in any medium or format, as long as you give appropriate credit to the original author(s) and the source, provide a link to the Creative Commons licence, and indicate if changes were made. The images or other third party material in this article are included in the article's Creative Commons licence, unless indicated otherwise in a credit line to the material. If material is not included in the article's Creative Commons licence and your intended use is not permitted by statutory regulation or exceeds the permitted use, you will need to obtain permission directly from the copyright holder. To view a copy of this licence, visit http://creativecommons.org/licenses/by/4.0/. 


\section{References}

1. Dunkelberger JR, Song WC (2010) Complement and its role in innate and adaptive immune responses. Cell Res 20(1):34-50

2. Java A, Apicelli AJ, Liszewski MK, Coler-Reilly A, Atkinson JP, Kim AH et al (2020) The complement system in COVID-19: friend and foe? JCI Insight. https://doi.org/10.1172/jci.insight. 140711

3. Ma L, Sahu SK, Cano M, Kuppuswamy V, Bajwa J, McPhatter JN et al (2021) Increased complement activation is a distinctive feature of severe SARS-CoV-2 infection. Sci Immunol 6(59):eabh2259

4. Magro C, Mulvey JJ, Berlin D, Nuovo G, Salvatore S, Harp J et al (2020) Complement associated microvascular injury and thrombosis in the pathogenesis of severe COVID-19 infection: A report of five cases. Transl Res 220:1-13

5. Gao T, Hu M, Zhang X, Li H, Zhu L, Liu H, et al. Highly pathogenic coronavirus $\mathrm{N}$ protein aggravates lung injury by MASP2-mediated complement over-activation. medRxiv. 2020; 25: 777

6. Ackermann M, Verleden SE, Kuehnel M, Haverich A, Welte $\mathrm{T}$, Laenger $\mathrm{F}$ et al (2020) Pulmonary vascular endothelialitis, thrombosis, and angiogenesis in Covid-19. N Engl J Med 383(2): $120-128$

7. Noris M, Benigni A, Remuzzi G (2020) The case of complement activation in COVID-19 multiorgan impact. Kidney Int 98(2):314-322

8. Tan CW, Tan JY, Wong WH, Cheong MA, Ng IM, Conceicao EP et al (2021) Clinical and laboratory features of hypercoagulability in COVID-19 and other respiratory viral infections amongst predominantly younger adults with few comorbidities. Sci Rep 11(1): 1793

9. Fan BE, Umapathi T, Chua K, Chia YW, Wong SW, Tan GWL et al (2021) Delayed catastrophic thrombotic events in young and asymptomatic post COVID-19 patients. J Thromb Thrombolysis 51(4):971-977

10. Campbell CM, Kahwash R (2020) Will complement inhibition be the new target in treating COVID-19-related systemic thrombosis? Circulation 141(22):1739-1741

11. Willems E, Alkema W, Keizer-Garritsen J, Suppers A, van der Flier M, Philipsen R et al (2019) Biosynthetic homeostasis and resilience of the complement system in health and infectious disease. EBioMedicine 45:303-313

12. Feng S, Liang X, Kroll MH, Chung DW, Afshar-Kharghan V (2015) von Willebrand factor is a cofactor in complement regulation. Blood 125(6):1034-1037

13. Bonaventura A, Vecchie A, Dagna L, Martinod K, Dixon DL, Van Tassell BW et al (2021) Endothelial dysfunction and immunothrombosis as key pathogenic mechanisms in COVID-19. Nat Rev Immunol 21(5):319-329

14. Mancini I, Baronciani L, Artoni A, Colpani P, Biganzoli M, Cozzi $\mathrm{G}$ et al (2021) The ADAMTS13-von Willebrand factor axis in COVID-19 patients. J Thromb Haemost 19(2):513-521

15. Horvath B, Hegedus D, Szapary L, Marton Z, Alexy T, Koltai K et al (2004) Measurement of von Willebrand factor as the marker of endothelial dysfunction in vascular diseases. Exp Clin Cardiol 9(1):31-34

16. Danwang C, Endomba FT, Nkeck JR, Wouna DLA, Robert A, Noubiap JJ (2020) A meta-analysis of potential biomarkers associated with severity of coronavirus disease 2019 (COVID-19). Biomark Res 8:37

17. Mahler M, Meroni PL, Infantino M, Buhler KA, Fritzler MJ (2021) Circulating calprotectin as a biomarker of COVID-19 severity. Expert Rev Clin Immunol 17(5):431-443

18. Garcia L, de Guadiana R, Mulero MDR, Olivo MH, Rojas CR, Arenas VR, Morales MG et al (2021) Circulating levels of
GDF-15 and calprotectin for prediction of in-hospital mortality in COVID-19 patients: A case series. J Infect. https://doi.org/10. 1016/j.jinf.2020.08.010

19. Shi H, Zuo Y, Yalavarthi S, Gockman K, Zuo M, Madison JA et al (2021) Neutrophil calprotectin identifies severe pulmonary disease in COVID-19. J Leukoc Biol 109(1):67-72

20. Ramlall V, Thangaraj PM, Meydan C, Foox J, Butler D, Kim $\mathrm{J}$ et al (2020) Immune complement and coagulation dysfunction in adverse outcomes of SARS-CoV-2 infection. Nat Med 26(10):1609-1615

21. Lupu F, Keshari RS, Lambris JD, Coggeshall KM (2014) Crosstalk between the coagulation and complement systems in sepsis. Thromb Res 133(Suppl 1):S28-31

22. Wool GD, Miller JL (2021) The impact of COVID-19 disease on platelets and coagulation. Pathobiology 88(1):15-27

23. Mannes M, Dopler A, Zolk O, Lang SJ, Halbgebauer R, Hochsmann B et al (2021) Complement inhibition at the level of C3 or C5: mechanistic reasons for ongoing terminal pathway activity. Blood 137(4):443-455

24. Distelmaier K, Adlbrecht C, Jakowitsch J, Winkler S, Dunkler $\mathrm{D}$, Gerner $\mathrm{C}$ et al (2009) Local complement activation triggers neutrophil recruitment to the site of thrombus formation in acute myocardial infarction. Thromb Haemost 102(3):564-572

25. Foley JH, Walton BL, Aleman MM, O'Byrne AM, Lei V, Harrasser $\mathrm{M}$ et al (2016) Complement activation in arterial and venous thrombosis is mediated by plasmin. EBioMedicine $5: 175-182$

26. Ito T, Kakuuchi M, Maruyama I (2021) Endotheliopathy in septic conditions: mechanistic insight into intravascular coagulation. Crit Care 25(1):95

27. Goshua G, Pine AB, Meizlish ML, Chang CH, Zhang H, Bahel P et al (2020) Endotheliopathy in COVID-19-associated coagulopathy: evidence from a single-centre, cross-sectional study. Lancet Haematol 7(8):e575-e582

28. Ladikou EE, Sivaloganathan H, Milne KM, Arter WE, Ramasamy $\mathrm{R}$, Saad R et al (2020) Von Willebrand factor (vWF): marker of endothelial damage and thrombotic risk in COVID-19? Clin Med (Lond) 20(5):e178-e182

29. Cugno M, Meroni PL, Gualtierotti R, Griffini S, Grovetti E, Torri A et al (2021) Complement activation and endothelial perturbation parallel COVID-19 severity and activity. J Autoimmun 116:102560

30. Yu J, Yuan X, Chen H, Chaturvedi S, Braunstein EM, Brodsky RA (2020) Direct activation of the alternative complement pathway by SARS-CoV-2 spike proteins is blocked by factor D inhibition. Blood 136(18):2080-2089

31. Duerschmied D, Suidan GL, Demers M, Herr N, Carbo C, Brill A et al (2013) Platelet serotonin promotes the recruitment of neutrophils to sites of acute inflammation in mice. Blood 121(6):1008-1015

32. Del Conde I, Cruz MA, Zhang H, Lopez JA, Afshar-Kharghan V (2005) Platelet activation leads to activation and propagation of the complement system. J Exp Med 201(6):871-879

33. Hottz ED, Azevedo-Quintanilha IG, Palhinha L, Teixeira L, Barreto EA, Pao CRR et al (2020) Platelet activation and plateletmonocyte aggregate formation trigger tissue factor expression in patients with severe COVID-19. Blood 136(11):1330-1341

34. Yao Y, Cao J, Wang Q, Shi Q, Liu K, Luo Z et al (2020) D-dimer as a biomarker for disease severity and mortality in COVID-19 patients: a case control study. J Intensive Care 8:49

35. Osawa I, Okamoto K, Ikeda M, Otani A, Wakimoto Y, Yamashita $M$ et al (2021) Dynamic changes in fibrinogen and D-dimer levels in COVID-19 patients on nafamostat mesylate. J Thromb Thrombolysis 51(3):649-656 
36. Wijarnpreecha K, Ungprasert P, Panjawatanan P, Harnois DM, Zaver HB, Ahmed A et al (2021) COVID-19 and liver injury: a meta-analysis. Eur J Gastroenterol Hepatol 33(7):990-995

37 Baj J, Karakula-Juchnowicz H, Teresinski G, Buszewicz G, Ciesielka M, Sitarz E et al (2020) COVID-19: specific and nonspecific clinical manifestations and symptoms: the current state of knowledge. J Clin Med 9(6):1753

38. Cuschieri S (2019) The CONSORT statement. Saudi J Anaesth 13(Suppl 1):S27-S30
Publisher's Note Springer Nature remains neutral with regard to jurisdictional claims in published maps and institutional affiliations. 